\title{
Thiadiazole Derivatives as Potential Anticonvulsant Agents
}

\author{
Pooja Mullick, Suroor A. Khan, Surajpal Verma, and Ozair Alam
Department of Pharmaceutical Chemistry, Faculty of Pharmacy, Jamia Hamdard (Hamdard University) Hamdard Nagar, New Delhi-110062, India. ${ }^{*}$ E-mail: poojamullick7@rediffmail.com
Received August 30, 2010, Accepted December 20, 2010

\begin{abstract}
A series of thiadiazole derivatives were synthesized with differently substituted benzoic acids which were cyclized to give differently substituted thiazolidin-4-one. Elemental analysis, IR, ${ }^{1} \mathrm{H}$ NMR, ${ }^{13} \mathrm{C}$ NMR and mass spectral data confirmed the structure of the synthesized compounds. The derivatives of these moieties were evaluated for anticonvulsant activity by MES model and neurotoxicity by rotarod method. The synthesized compounds showed good potential for anticonvulsant activity besides this, the compounds also showed neurotoxic effect. It was observed that compounds with $\mathrm{OCH}_{3}$ at 3, 4 position of phenyl ring [5(a-l)] showed less protection against convulsions as compared to compounds having unsubstituted phenyl ring $[\mathbf{4}(\mathbf{a}-\mathbf{l})]$.
\end{abstract}

Key Words : Thiadiazole, Thiazolidin-4-one, Anticonvulsant activity, Neurotoxicity

\section{Introduction}

Current drug therapy for epilepsy includes Phenytoin, Carbamazepine, Lamotrigine etc. The main disadvantage with the current therapy is significant side effects which may limit their use. In recent years, anti-epileptic drug development has been one of the most prominent research areas with the in-depth understanding of the pathophysiology of epilepsy. Pandeya ${ }^{1}$ has proposed the identifiable features for anticonvulsant activity as (i) hydrophobic aryl ring (Ar), (ii) a hydrogen bonding domain (HBD), (iii) an electron-donor group (D) and (iv) another distal hydrophobic site which are evident in the existing anticonvulsant drugs.

Following above hypothesis, in the present study we synthesized the final compounds as 5-benzylidene-2-[\{5-(substituted phenyl)-[1,3,4]-thiadiazol-2-yl\}imino]-1,3-thiazolidin-4-one 4(a-l) and 2-[\{5-(substituted phenyl)-[1,3,4]-thiadiazol-2-yl $\}$ imino]-5-(3,4-dimethoxybenzylidene)-1,3-thiazolidin-4-one 5(a-I) which posses two aromatic rings as hydrophobic domain, $\mathrm{N}=\mathrm{C}-\mathrm{NH}$ exist as hydrogen bonding domain and carbonyl group is the electron donor group. According to the above hypothesis, all these are the important structural features responsible for compounds to show anti-convulsant activity. And hence, prompted us to envisage anti-convulsant and neurotoxicity activity for the mentioned compounds.

\section{Experimental}

General Method for Synthesis of 2-Amino-5-(substituted phenyl)-(1,3,4)-thiadiazole 1(a-l). A mixture of $40 \mathrm{mmol}$ of differently substituted benzoic acids and the equimolar amount of thiosemicarbazide and phosphorous oxychloride $(30 \mathrm{~mL})$ was refluxed gently for 2 - $4 \mathrm{hrs}$. The reaction mixture was allowed to cool; ice cold water $(100 \mathrm{~mL})$ was added to the flask. The mixture was again set for refluxing for about $4 \mathrm{hrs}$. and filtered. The solution was neutralized with ammonia solution. The precipitate was filtered, washed with water, dried and recrystallized from ethanol-water to yield compounds $\mathbf{1}(\mathbf{a}-\mathbf{l})$. The purity of compounds was analyzed by TLC using benzene: acetone (9:1) as mobile phase.

2-Amino-5-phenyl-(1,3,4)-thiadiazole 1(a): $\operatorname{mp} 224-225^{\circ} \mathrm{C}$; \%Yield: 71; IR (KBr) cm $\mathrm{cm}^{-1}: 3496(\mathrm{NH}), 1595(\mathrm{C}=\mathrm{N}), 1604$ $(\mathrm{C}=\mathrm{C}) ;{ }^{1} \mathrm{H}$ NMR $\left(300 \mathrm{MHz}, \mathrm{DMSO}-d_{6}\right) \delta$ 6.64-7.19 (m, 5H, $\mathrm{ArH}), 12.04$ (s, 2H, NH${ }_{2}$ ).

2-Amino-5-(2-methylphenyl)-(1,3,4)-thiadiazole 1(b): $\mathrm{mp}$ $210-211^{\circ} \mathrm{C}$; \%Yield: 73; IR (KBr) cm $\mathrm{cm}^{-1}: 3446(\mathrm{NH}), 1593$ $(\mathrm{C}=\mathrm{N}), 1636(\mathrm{C}=\mathrm{C}) ;{ }^{1} \mathrm{H}$ NMR $\left(300 \mathrm{MHz}, \mathrm{DMSO}-d_{6}\right) \delta 2.33$ (s, 3H, $\left.\mathrm{CH}_{3}\right), 6.64-7.19$ (m, 4H, ArH), 12.04 (s, 2H, $\mathrm{NH}_{2}$ ).

2-Amino-5-(4-methylphenyl)-(1,3,4)-thiadiazole 1(c): $\mathrm{mp}$ 215 - $216^{\circ} \mathrm{C}$; \% Yield: 79; IR (KBr) cm ${ }^{-1}: 3477\left(\mathrm{NH}_{2}\right), 1587$ $(\mathrm{C}=\mathrm{N}), 1644(\mathrm{C}=\mathrm{C}) ;{ }^{1} \mathrm{H}$ NMR (300 MHz, DMSO- $\left.d_{6}\right) \delta 2.35$ (s, 3H, $\left.\mathrm{CH}_{3}\right), 6.71-7.20$ (m, 4H, ArH), 12.15 (s, 2H, $\mathrm{NH}_{2}$ ).

2-Amino-5-(2-Chlorophenyl)-(1,3,4)-thiadiazole 1(d): $\mathrm{mp}$ 204 - $206{ }^{\circ} \mathrm{C}$; \%Yield: 67; IR (KBr) cm $\mathrm{cm}^{-1}: 3465(\mathrm{NH}), 1557$ $(\mathrm{C}=\mathrm{N}), 1614(\mathrm{C}=\mathrm{C}) ;{ }^{1} \mathrm{H}$ NMR $\left(300 \mathrm{MHz}, \mathrm{DMSO}-d_{6}\right) \delta 6.78-$ 7.24 (m, 4H, ArH), 12.04 (s, 2H, $\left.\mathrm{NH}_{2}\right)$.

2-Amino-5-(4-chlorophenyl)-(1,3,4)-thiadiazole 1(e): $\mathrm{mp}$ 230 - $232{ }^{\circ} \mathrm{C}$; \% Yield: 77; IR (KBr) cm ${ }^{-1}: 3468(\mathrm{NH}), 1572$ $(\mathrm{C}=\mathrm{N}), 1594(\mathrm{C}=\mathrm{C}) ;{ }^{1} \mathrm{H}$ NMR $\left(300 \mathrm{MHz}, \mathrm{DMSO}-d_{6}\right) \delta 6.86-$ 7.25 (m, 4H, ArH), 11.64 (s, 2H, $\left.\mathrm{NH}_{2}\right)$.

2-Amino-5-(2-bromophenyl)-(1,3,4)-thiadiazole 1(f): $\mathrm{mp}$ 192 - $194{ }^{\circ} \mathrm{C}$; \% Yield: 62; IR (KBr) cm ${ }^{-1}: 3492(\mathrm{NH}), 1583$ $(\mathrm{C}=\mathrm{N}), 1619(\mathrm{C}=\mathrm{C}) ;{ }^{1} \mathrm{H}$ NMR $\left(300 \mathrm{MHz}, \mathrm{DMSO}-d_{6}\right) \delta 6.84-$ 7.17 (m, 4H, ArH), $12.16\left(\mathrm{~s}, 2 \mathrm{H}, \mathrm{NH}_{2}\right)$.

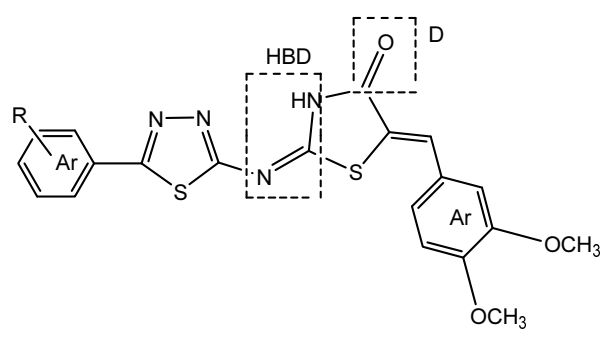

Figure 1. Synthesized compound representing structural similarity to anti-convulsants. 
2-Amino-5-(4-bromophenyl)-(1,3,4)-thiadiazole 1(g): mp 247 - $248{ }^{\circ} \mathrm{C}$; \%Yield: 61; IR (KBr) cm ${ }^{-1}: 3396(\mathrm{NH}), 1569$ $(\mathrm{C}=\mathrm{N}), 1582(\mathrm{C}=\mathrm{C}) ;{ }^{1} \mathrm{H}$ NMR $\left(300 \mathrm{MHz}, \mathrm{DMSO}-d_{6}\right) \delta 6.64-$ 7.19 (m, 4H, ArH), 12.07 (s, 2H, NH$)$.

2-Amino-5-(3-nitrophenyl)-(1,3,4)-thiadiazole 1(h): mp 236 $238^{\circ} \mathrm{C}$; \% Yield: 66; IR $(\mathrm{KBr}) \mathrm{cm}^{-1}: 3426(\mathrm{NH}), 1564(\mathrm{C}=\mathrm{N})$, $1558(\mathrm{C}=\mathrm{C}) ;{ }^{1} \mathrm{H}$ NMR $\left(300 \mathrm{MHz}, \mathrm{DMSO}-d_{6}\right) \delta 6.88-7.23(\mathrm{~m}$, $4 \mathrm{H}, \mathrm{ArH}), 12.24\left(\mathrm{~s}, 2 \mathrm{H}, \mathrm{NH}_{2}\right)$.

2-Amino-5-(4-nitrophenyl)-(1,3,4)-thiadiazole 1(i): mp 227 $229^{\circ} \mathrm{C}$; \% Yield: 60; IR (KBr) cm ${ }^{-1}: 3452(\mathrm{NH}), 1536(\mathrm{C}=\mathrm{N})$, $1548(\mathrm{C}=\mathrm{C}) ;{ }^{1} \mathrm{H}$ NMR $\left(300 \mathrm{MHz}, \mathrm{DMSO}-d_{6}\right) \delta 6.88-7.24(\mathrm{~m}$, $4 \mathrm{H}, \mathrm{ArH}), 12.35\left(\mathrm{~s}, 2 \mathrm{H}, \mathrm{NH}_{2}\right)$.

2-Amino-5-(2,4-dichlorophenyl)-(1,3,4)-thiadiazole 1(j): mp 240 - $242{ }^{\circ} \mathrm{C}$; \% Yield: 82; IR (KBr) cm ${ }^{-1}$ : $3506(\mathrm{NH}), 1598$ $(\mathrm{C}=\mathrm{N}), 1579(\mathrm{C}=\mathrm{C}) ;{ }^{1} \mathrm{H}$ NMR $\left(300 \mathrm{MHz}, \mathrm{DMSO}-d_{6}\right) \delta 7.14-$ $7.30(\mathrm{~m}, 3 \mathrm{H}, \mathrm{ArH}), 12.34$ (s, 2H, $\left.\mathrm{NH}_{2}\right)$.

2-Amino-5-(2-hydroxyphenyl)-(1,3,4)-thiadiazole 1(k): $\mathrm{mp}$ 184 - $185{ }^{\circ} \mathrm{C}$; \%Yield: 73; IR (KBr) cm $\mathrm{cm}^{-1}: 3493(\mathrm{NH}), 1575$ $(\mathrm{C}=\mathrm{N}), 1549(\mathrm{C}=\mathrm{C}) ;{ }^{1} \mathrm{H}$ NMR $\left(300 \mathrm{MHz}, \mathrm{DMSO}-d_{6}\right) \delta 6.58-$ $6.93(\mathrm{~m}, 4 \mathrm{H}, \mathrm{ArH}), 9.42(\mathrm{~s}, 1 \mathrm{H}, \mathrm{OH}), 11.56\left(\mathrm{~s}, 2 \mathrm{H}, \mathrm{NH}_{2}\right)$.

2-Amino-5-(4-methoxyphenyl)-(1,3,4)-thiadiazole 1(l): $\mathrm{mp}$ 209 - $210{ }^{\circ} \mathrm{C}$; \% Yield: 78; IR (KBr) cm $\mathrm{cm}^{-1}: 3426(\mathrm{NH}), 1555$ $(\mathrm{C}=\mathrm{N}), 1618(\mathrm{C}=\mathrm{C}) ;{ }^{1} \mathrm{H}$ NMR $\left(300 \mathrm{MHz}, \mathrm{DMSO}-d_{6}\right) \delta 3.16$ (s, 3H, $\left.\mathrm{OCH}_{3}\right), 6.64-7.19$ (m, 4H, ArH), 11.46 (s, 2H, $\mathrm{NH}_{2}$ ).

General Method forSynthesis of 2-chloro- $\mathrm{N}$-[5-(Substituted phenyl)-[1,3,4]-thiadiazol-2-yl]-acetamide 2(a-l). To the mixture of appropriately substituted compound 1(a-l) $(10 \mathrm{mmol})$ in $15 \mathrm{~mL}$ of dry benzene and $2 \mathrm{~mL}$ of dry pyridine, chloroacetylchloride $(20 \mathrm{mmol})$ in $10 \mathrm{~mL}$ of dry benzene was added drop wise with a constant stirring at room temperature. After complete addition, the reaction mixture was refluxed for about 6 $8 \mathrm{hrs}$. and poured over crushed ice. The precipitate was filtered, washed with water, dried and recrystallized from dioxane-water to yield compound 2(a-l). The purity of compounds was analyzed by TLC using benzene: acetone (9:1) as mobile phase.

2-Chloro- $\boldsymbol{N}$-[5-phenyl-[1,3,4]-thiadiazol-2-yl]-acetamide 2(a): $\mathrm{mp} 228$ - $229^{\circ} \mathrm{C}$; \% Yield: 87; IR (KBr) cm ${ }^{-1}: 3130(\mathrm{NH})$, $1695(\mathrm{C}=\mathrm{O}), 1572(\mathrm{C}=\mathrm{N}), 764(\mathrm{C}-\mathrm{Cl}) ;{ }^{1} \mathrm{H}$ NMR $(300 \mathrm{MHz}$, DMSO- $\left.d_{6}\right) \delta 4.48\left(\mathrm{~s}, 2 \mathrm{H}, \mathrm{CH}_{2}\right), 7.54-7.96(\mathrm{~m}, 5 \mathrm{H}, \mathrm{ArH})$, 13.07 (bs, $1 \mathrm{H}, \mathrm{NH}) ;{ }^{13} \mathrm{C}$ NMR $\left(75 \mathrm{MHz}, \mathrm{DMSO}-d_{6}\right) \delta 48.6$, 126.7, 127.1, 128.6, 129.0, 129.4, 136.5, 154.2, 161.5, 164.2; FAB-MS $m / z: 254(\mathrm{M})^{+}, 256(\mathrm{M}+2)^{+}$.

2-Chloro- $\mathrm{N}$-[5-(2-methylphenyl)-[1,3,4]-thiadiazol-2-yl]acetamide 2(b): $\mathrm{mp} 213-215^{\circ} \mathrm{C}$; \% Yield: 82 ; IR $(\mathrm{KBr}) \mathrm{cm}^{-1}$ : $3340(\mathrm{NH}), 1648(\mathrm{C}=\mathrm{O}), 1552(\mathrm{C}=\mathrm{N}), 761(\mathrm{C}-\mathrm{Cl}) ;{ }^{1} \mathrm{H}$ NMR (300 MHz, DMSO- $\left.d_{6}\right) \delta 2.34\left(\mathrm{~s}, 3 \mathrm{H}, \mathrm{CH}_{3}\right), 4.28\left(\mathrm{~s}, 2 \mathrm{H}, \mathrm{CH}_{2}\right)$, 7.52-7.87 (m, 4H, ArH), 12.94 (bs, 1H, NH).

2-Chloro- $\mathrm{N}$-[5-(4-methylphenyl)-[1,3,4]-thiadiazol-2-yl]acetamide 2(c): $\mathrm{mp} 238-240{ }^{\circ} \mathrm{C}$; \% Yield: 87 ; IR (KBr) $\mathrm{cm}^{-1}$ : $3160(\mathrm{NH}), 1653(\mathrm{C}=\mathrm{O}), 1574(\mathrm{C}=\mathrm{N}), 766(\mathrm{C}-\mathrm{Cl}) ;{ }^{1} \mathrm{H}$ NMR $\left(300 \mathrm{MHz}, \mathrm{DMSO}-d_{6}\right) \delta 2.36\left(\mathrm{~s}, 3 \mathrm{H}, \mathrm{CH}_{3}\right), 4.31\left(\mathrm{~s}, 2 \mathrm{H}, \mathrm{CH}_{2}\right)$, 7.53-7.84 (m, 4H, ArH), 12.64 (bs, 1H, NH).

2-Chloro- $N$-[5-(2-chlorophenyl)-[1,3,4]-thiadiazol-2-yl]acetamide 2(d): $\mathrm{mp} 229-230^{\circ} \mathrm{C}$; \% Yield: 86 ; IR $(\mathrm{KBr}) \mathrm{cm}^{-1}$ : $3364(\mathrm{NH}), 1687(\mathrm{C}=\mathrm{O}), 1535(\mathrm{C}=\mathrm{N}), 757(\mathrm{C}-\mathrm{Cl}) ;{ }^{1} \mathrm{H}$ NMR $\left(300 \mathrm{MHz}, \mathrm{DMSO}-d_{6}\right) \delta 4.29\left(\mathrm{~s}, 2 \mathrm{H}, \mathrm{CH}_{2}\right), 7.58-7.91(\mathrm{~m}, 4 \mathrm{H}$, $\mathrm{ArH}), 12.65$ (bs, 1H, NH).

2-Chloro- $N$-[5-(4-chlorophenyl)-[1,3,4]-thiadiazol-2-yl]- acetamide 2(e): $\mathrm{mp} 243-244{ }^{\circ} \mathrm{C} ; \%$ Yield: 90; IR (KBr) $\mathrm{cm}^{-1}$ : $3230(\mathrm{NH}), 1686(\mathrm{C}=\mathrm{O}), 1554(\mathrm{C}=\mathrm{N}), 759(\mathrm{C}-\mathrm{Cl}) ;{ }^{1} \mathrm{H}$ NMR $\left(300 \mathrm{MHz}, \mathrm{DMSO}-d_{6}\right) \delta 4.32\left(\mathrm{~s}, 2 \mathrm{H}, \mathrm{CH}_{2}\right), 7.53-7.87$ (m, 4H, $\mathrm{ArH}), 13.18$ (bs,1H,NH).

2-Chloro- $\mathrm{N}$-[5-(2-bromophenyl)-[1,3,4]-thiadiazol-2-yl]acetamide 2(f): $\mathrm{mp} 263-265^{\circ} \mathrm{C}$; \% Yield: 69 ; IR $(\mathrm{KBr}) \mathrm{cm}^{-1}$ : $3275(\mathrm{NH}), 1646(\mathrm{C}=\mathrm{O}), 1552(\mathrm{C}=\mathrm{N}), 754(\mathrm{C}-\mathrm{Cl}) ;{ }^{1} \mathrm{H}$ NMR $\left(300 \mathrm{MHz}, \mathrm{DMSO}-d_{6}\right) \delta 4.37\left(\mathrm{~s}, 2 \mathrm{H}, \mathrm{CH}_{2}\right), 7.52-7.84(\mathrm{~m}, 4 \mathrm{H}$, ArH), 12.73 (bs, 1H, NH).

2-Chloro- $\mathrm{N}$-[5-(4-bromophenyl)-[1,3,4]-thiadiazol-2-yl]acetamide 2(g): $\mathrm{mp} 241-242{ }^{\circ} \mathrm{C}$; \% Yield: 63 ; IR $(\mathrm{KBr}) \mathrm{cm}^{-1}$ : $3264(\mathrm{NH}), 1665(\mathrm{C}=\mathrm{O}), 1567(\mathrm{C}=\mathrm{N}), 762(\mathrm{C}-\mathrm{Cl}) ;{ }^{1} \mathrm{H}$ NMR $\left(300 \mathrm{MHz}, \mathrm{DMSO}-d_{6}\right) \delta 4.42\left(\mathrm{~s}, 2 \mathrm{H}, \mathrm{CH}_{2}\right), 7.67-7.91(\mathrm{~m}, 4 \mathrm{H}$, ArH), 11.86 (bs, 1H, NH).

2-Chloro- $N$-[5-(3-nitrophenyl)-[1,3,4]-thiadiazol-2-yl]acetamide 2(h): $\mathrm{mp} 168-170^{\circ} \mathrm{C}$; \% Yield: 64 ; IR $(\mathrm{KBr}) \mathrm{cm}^{-1}$ : $3374(\mathrm{NH}), 1673(\mathrm{C}=\mathrm{O}), 1577(\mathrm{C}=\mathrm{N}), 751(\mathrm{C}-\mathrm{Cl}) ;{ }^{1} \mathrm{H}$ NMR $\left(300 \mathrm{MHz}, \mathrm{DMSO}-d_{6}\right) \delta 4.27\left(\mathrm{~s}, 2 \mathrm{H}, \mathrm{CH}_{2}\right), 7.58-7.82(\mathrm{~m}, 4 \mathrm{H}$, ArH), 12.74 (bs, 1H, NH).

2-Chloro- $N$-[5-(4-nitrophenyl)-[1,3,4]-thiadiazol-2-yl]acetamide 2(i): $\mathrm{mp} 162-163{ }^{\circ} \mathrm{C}$; \% Yield: 67 ; IR $(\mathrm{KBr}) \mathrm{cm}^{-1}$ : $3266(\mathrm{NH}), 1646(\mathrm{C}=\mathrm{O}), 1554(\mathrm{C}=\mathrm{N}), 755(\mathrm{C}-\mathrm{Cl}) ;{ }^{1} \mathrm{H}$ NMR $\left(300 \mathrm{MHz}, \mathrm{DMSO}-d_{6}\right) \delta 4.25\left(\mathrm{~s}, 2 \mathrm{H}, \mathrm{CH}_{2}\right), 7.54-7.81(\mathrm{~m}, 4 \mathrm{H}$, ArH), 11.53 (bs, 1H, NH).

2-Chloro- $N$-[5-(2,4-dichlorophenyl)-[1,3,4]-thiadiazol-2yl]-acetamide 2(j): $\mathrm{mp} 246-247^{\circ} \mathrm{C}$; \% Yield: 82 ; IR (KBr) cm ${ }^{-1}$ : $3276(\mathrm{NH}), 1657(\mathrm{C}=\mathrm{O}), 1576(\mathrm{C}=\mathrm{N}), 759(\mathrm{C}-\mathrm{Cl}) ;{ }^{1} \mathrm{H}$ NMR $\left(300 \mathrm{MHz}, \mathrm{DMSO}-d_{6}\right) \delta 4.46\left(\mathrm{~s}, 2 \mathrm{H}, \mathrm{CH}_{2}\right), 7.63-7.79(\mathrm{~m}, 3 \mathrm{H}$, $\mathrm{ArH}), 12.57$ (bs, 1H, NH).

2-Chloro- $\mathrm{N}$-[5-(2-hydroxyphenyl)-[1,3,4]-thiadiazol-2-yl]acetamide 2(k): $\mathrm{mp} 203-205^{\circ} \mathrm{C}$; \% Yield: 79; IR (KBr) $\mathrm{cm}^{-1}$ : $3267(\mathrm{NH}), 1583(\mathrm{C}=\mathrm{O}), 1524(\mathrm{C}=\mathrm{N}), 751(\mathrm{C}-\mathrm{Cl}) ;{ }^{1} \mathrm{H}$ NMR $\left(300 \mathrm{MHz}, \mathrm{DMSO}-d_{6}\right) \delta 4.23\left(\mathrm{~s}, 2 \mathrm{H}, \mathrm{CH}_{2}\right), 7.43-7.66(\mathrm{~m}, 4 \mathrm{H}$, ArH), 9.47 (s, 1H, OH), 12.37 (bs, 1H, NH).

2-Chloro- $\mathrm{N}$-[5-(4-methoxyphenyl)-[1,3,4]-thiadiazol-2-yl]acetamide 2(I): $\mathrm{mp} 231-232{ }^{\circ} \mathrm{C}$; \% Yield: 87 ; IR $(\mathrm{KBr}) \mathrm{cm}^{-1}$ : $3370(\mathrm{NH}), 1637(\mathrm{C}=\mathrm{O}), 1576(\mathrm{C}=\mathrm{N}), 757(\mathrm{C}-\mathrm{Cl}) ;{ }^{1} \mathrm{H}$ NMR $\left(300 \mathrm{MHz}, \mathrm{DMSO}-d_{6}\right) \delta 3.43\left(\mathrm{~s}, 3 \mathrm{H}, \mathrm{OCH}_{3}\right), 4.26\left(\mathrm{~s}, 2 \mathrm{H}, \mathrm{CH}_{2}\right)$, 7.52-7.71 (m, 4H, ArH), 11.50 (bs, 1H, NH).

General Method for Synthesis of 2-[ 5 5-(Substituted phenyl)$[1,3,4]$-thiadiazol-2-yl\} imino]-1,3-thiazolidin-4-one 3(a-l). 7 mmol of each compound 2(a-l) and ammonium thiocyanate $(15 \mathrm{mmol}$ ) in $35 \mathrm{~mL}$ ethanol was refluxed for $3 \mathrm{hrs}$, hold reaction mixture overnight. The product obtained was filtered, dried and recrystallized from ethanol-water to yield compound $3(\mathbf{a}-\mathbf{l})$. The purity of compounds was analyzed by TLC using benzene: acetone $(9: 1)$ as mobile phase.

2-[\{5-Phenyl-[1,3,4]-thiadiazol-2-yl\} imino]-1,3-thiazolidin4-one 3(a): $\mathrm{mp} 128$ - $130{ }^{\circ} \mathrm{C}$; \% Yield: 76; IR (KBr) cm ${ }^{-1}: 3374$ $(\mathrm{NH}), 1762(\mathrm{C}=\mathrm{O}), 1592(\mathrm{C}=\mathrm{N}) ;{ }^{1} \mathrm{H}$ NMR $(300 \mathrm{MHz}$, DMSO- $\left.d_{6}\right) \delta 4.13$ (s, 2H, $\left.\mathrm{CH}_{2}-\mathrm{CO}\right), 7.52-7.75$ (m, 5H, ArH), 12.38 (bs, 1H, NH, D 2 -exchangeble); ${ }^{13} \mathrm{C}$ NMR $(75 \mathrm{MHz}$, DMSO- $\left.d_{6}\right) \delta 37.4,126.4,127.7,128.3,129.0,129.5,136.7$, 154.1, 162.6, 163.8, 173.4; FAB-MS $m / z: 276(\mathrm{M})^{+}$.

2-[\{5-(2-Methylphenyl)-[1,3,4]-thiadiazol-2-yl\} imino]-1,3thiazolidin-4-one 3(b): $\mathrm{mp} 133-135^{\circ} \mathrm{C}$; \% Yield: 72; IR (KBr) $\mathrm{cm}^{-1}$ : $3385(\mathrm{NH}), 1775(\mathrm{C}=\mathrm{O}), 1575(\mathrm{C}=\mathrm{N}) ;{ }^{1} \mathrm{H}$ NMR (300 $\left.\mathrm{MHz}, \mathrm{DMSO}-d_{6}\right) \delta 2.36\left(\mathrm{~s}, 3 \mathrm{H}, \mathrm{CH}_{3}\right), 4.08$ (s, 2H, $\left.\mathrm{CH}_{2}-\mathrm{CO}\right)$, 
7.42- 7.58 (m, 4H, ArH), 12.18 (bs, 1H, NH).

2-[\{5-(4-Methylphenyl)-[1,3,4]-thiadiazol-2-yl\} imino]-1,3thiazolidin-4-one 3(c): $\mathrm{mp} 122-123{ }^{\circ} \mathrm{C}$; \% Yield: 83; IR (KBr) $\mathrm{cm}^{-1}$ : $3402(\mathrm{NH}), 1757(\mathrm{C}=\mathrm{O}), 1587(\mathrm{C}=\mathrm{N}) ;{ }^{1} \mathrm{H}$ NMR $(300$ MHz, DMSO- $\left.d_{6}\right) \delta 2.32$ (s, 3H, $\left.\mathrm{CH}_{3}\right), 4.13$ (s, 2H, $\left.\mathrm{CH}_{2}-\mathrm{CO}\right)$, 7.49-7.63 (m, 4H, ArH), 11.85 (bs, 1H, NH).

2-[\{5-(2-Chlorophenyl)-[1,3,4]-thiadiazol-2-yl\} imino]-1,3thiazolidin-4-one 3(d): $\mathrm{mp} 162-164^{\circ} \mathrm{C}$; \% Yield: 71; IR (KBr) $\mathrm{cm}^{-1}$ : $3418(\mathrm{NH}), 1735(\mathrm{C}=\mathrm{O}), 1592(\mathrm{C}=\mathrm{N}) ;{ }^{1} \mathrm{H}$ NMR $(300$ $\left.\mathrm{MHz}, \mathrm{DMSO}-d_{6}\right) \delta 4.51$ (s, 2H, $\left.\mathrm{CH}_{2}-\mathrm{CO}\right), 7.61-7.74(\mathrm{~m}, 4 \mathrm{H}$, ArH), 13.08 (bs, 1H, NH).

2-[\{5-(4-Chlorophenyl)-[1,3,4]-thiadiazol-2-yl\} imino]-1,3thiazolidin-4-one 3(e): $\mathrm{mp} 174-177^{\circ} \mathrm{C}$; \% Yield: 78; IR (KBr) $\mathrm{cm}^{-1}: 3428(\mathrm{NH}), 1751(\mathrm{C}=\mathrm{O}), 1593(\mathrm{C}=\mathrm{N}) ;{ }^{1} \mathrm{H} \mathrm{NMR}(300 \mathrm{MHz}$, DMSO- $\left.d_{6}\right) \delta 3.97$ (s, 2H, $\left.\mathrm{CH}_{2}-\mathrm{CO}\right), 7.63-7.78$ (m, 4H, ArH), 12.86 (bs, $1 \mathrm{H}, \mathrm{NH})$.

2-[\{5-(2-Bromophenyl)-[1,3,4]-thiadiazol-2-yl\} imino]-1,3thiazolidin-4-one 3(f): $\mathrm{mp} 117-119^{\circ} \mathrm{C}$; \% Yield: 69; IR (KBr) $\mathrm{cm}^{-1}$ : $3391(\mathrm{NH}), 1695(\mathrm{C}=\mathrm{O}), 1587(\mathrm{C}=\mathrm{N}) ;{ }^{1} \mathrm{H}$ NMR $(300$ $\left.\mathrm{MHz}, \mathrm{DMSO}-d_{6}\right) \delta 4.31$ (s, 2H, $\left.\mathrm{CH}_{2}-\mathrm{CO}\right), 7.63-7.77(\mathrm{~m}, 4 \mathrm{H}$, $\mathrm{ArH}), 12.89$ (bs, 1H, NH).

2-[\{5-(4-Bromophenyl)-[1,3,4]-thiadiazol-2-yl\} imino]-1,3thiazolidin-4-one 3(g): $\mathrm{mp} 135-136{ }^{\circ} \mathrm{C}$; \% Yield: 64; IR (KBr) $\mathrm{cm}^{-1}$ : $3386(\mathrm{NH}), 1705(\mathrm{C}=\mathrm{O}), 1582(\mathrm{C}=\mathrm{N}) ;{ }^{1} \mathrm{H}$ NMR $(300$ MHz, DMSO- $\left.d_{6}\right) \delta 4.27$ (s, 2H, $\left.\mathrm{CH}_{2}-\mathrm{CO}\right), 7.66-7.83(\mathrm{~m}, 4 \mathrm{H}$, ArH), 11.93 (bs, 1H, NH).

2-[\{5-(3-Nitrophenyl)-[1,3,4]-thiadiazol-2-yl\} imino]-1,3thiazolidin-4-one 3(h): $\mathrm{mp} 107-110{ }^{\circ} \mathrm{C}$; \% Yield: 54; IR (KBr) $\mathrm{cm}^{-1}$ : $3372(\mathrm{NH}), 1696(\mathrm{C}=\mathrm{O}), 1597(\mathrm{C}=\mathrm{N}) ;{ }^{1} \mathrm{H} \mathrm{NMR}(300 \mathrm{MHz}$, DMSO- $\left.d_{6}\right) \delta 4.11$ (s, 2H, CH$\left._{2}-\mathrm{CO}\right), 7.37-7.53$ (m, 4H, ArH), 13.03 (bs, 1H, NH).

2-[\{5-(4-Nitrophenyl)-[1,3,4]-thiadiazol-2-yl\} imino]-1,3thiazolidin-4-one 3(i): $\mathrm{mp} 146-148^{\circ} \mathrm{C}$; \% Yield: 57; IR (KBr) $\mathrm{cm}^{-1}$ : $3418(\mathrm{NH}), 1735(\mathrm{C}=\mathrm{O}), 1592(\mathrm{C}=\mathrm{N}) ;{ }^{1} \mathrm{H}$ NMR $(300$ $\left.\mathrm{MHz}, \mathrm{DMSO}-d_{6}\right) \delta 3.84$ (s, 2H, $\left.\mathrm{CH}_{2}-\mathrm{CO}\right), 7.37-7.54(\mathrm{~m}, 4 \mathrm{H}$, ArH), 12.73 (bs, 1H, NH).

2-[\{5-(2,4-Dichlorophenyl)-[1,3,4]-thiadiazol-2-yl\} imino]1,3-thiazolidin-4-one 3(j): mp $186-188^{\circ} \mathrm{C}$; \%Yield: 70; IR $(\mathrm{KBr}) \mathrm{cm}^{-1}: 3425(\mathrm{NH}), 1684(\mathrm{C}=\mathrm{O}), 1583(\mathrm{C}=\mathrm{N}) ;{ }^{1} \mathrm{H}$ NMR $\left(300 \mathrm{MHz}, \mathrm{DMSO}-d_{6}\right) \delta 4.37$ (s, 2H, $\left.\mathrm{CH}_{2}-\mathrm{CO}\right), 7.73-7.81(\mathrm{~m}$, $3 \mathrm{H}, \mathrm{ArH}), 12.38$ (bs, 1H, NH).

2-[\{5-(2-Hydroxyphenyl)-[1,3,4]-thiadiazol-2-yl\} imino]1,3-thiazolidin-4-one 3(k): mp $160-161^{\circ} \mathrm{C}$; \% Yield: 73; IR $(\mathrm{KBr}) \mathrm{cm}^{-1}$ : $3288(\mathrm{NH}), 1696(\mathrm{C}=\mathrm{O}), 1589(\mathrm{C}=\mathrm{N}) ;{ }^{1} \mathrm{H}$ NMR $\left(300 \mathrm{MHz}, \mathrm{DMSO}-d_{6}\right) \delta 3.91\left(\mathrm{~s}, 2 \mathrm{H}, \mathrm{CH}_{2}-\mathrm{CO}\right), 7.41-7.59(\mathrm{~m}$, $4 \mathrm{H}, \mathrm{ArH}), 9.43$ (s, 1H, OH), 11.76 (bs, 1 H, NH).

2-[\{5-(4-Methoxyphenyl)-[1,3,4]-thiadiazol-2-yl\} imino]1,3-thiazolidin-4-one 3(l): mp $108-110^{\circ} \mathrm{C}$; \%Yield: 82; IR $(\mathrm{KBr}) \mathrm{cm}^{-1}$ : $3428(\mathrm{NH}), 1735(\mathrm{C}=\mathrm{O}), 1591(\mathrm{C}=\mathrm{N}) ;{ }^{1} \mathrm{H}$ NMR $\left(300 \mathrm{MHz}, \mathrm{DMSO}-d_{6}\right) \delta 3.64\left(\mathrm{~s}, 3 \mathrm{H}, \mathrm{OCH}_{3}\right), 4.36(\mathrm{~s}, 2 \mathrm{H}$, $\mathrm{CH}_{2}$ - CO), 7.37-7.51 (m, 4H, ArH), 12.54 (bs, 1H, NH).

General Method for Synthesis of 5-Benzylidene-2-[\{5-(substituted phenyl)-[1,3,4]-thiadiazol-2-yl\} imino]-1,3-thiazolidin4-one 4(a-l). To compound 3(a-l) (3 mmol) in $20 \mathrm{~mL}$ ethanol, $2 \mathrm{~mL}$ piperidine and $3 \mathrm{mmol}$ of benzaldehyde was added. The mixture was refluxed for $12-18 \mathrm{hrs}$. The mixture was poured over crushed ice and the solution was neutralized with $\mathrm{HCl}$. The precipitate was filtered, washed with water, dried and re- crystallized from ethanol-DMF to yield final compound 4(a-l). The purity of compounds was analyzed by TLC using benzene: acetone $(9: 1)$ as mobile phase.

5-Benzylidene-2-[\{5-phenyl-[1,3,4]-thiadiazol-2-yl $\}$ imino]1,3-thiazolidin-4-one 4(a): $\mathrm{mp} 88$ - $90{ }^{\circ} \mathrm{C}$; \% Yield: 73; IR (KBr) $\mathrm{cm}^{-1}$ : $3328(\mathrm{NH}), 1758(\mathrm{C}=\mathrm{O}), 1593(\mathrm{C}=\mathrm{N}) ;{ }^{1} \mathrm{H}$ NMR (300 $\left.\mathrm{MHz}, \mathrm{DMSO}-d_{6}\right) \delta$ 7.21-7.53 (m, 1H-CH, 10H-ArH), 12.06 (bs, $1 \mathrm{H}, \mathrm{NH}, \mathrm{D}_{2} \mathrm{O}$-exchangeble); ${ }^{13} \mathrm{C} \mathrm{NMR}\left(75 \mathrm{MHz}, \mathrm{DMSO}-d_{6}\right)$ $\delta 120.7,125.9,126.4,126.8,127.1,127.4,129.4,130.3,136.5$, $142.5,154.3,162.9,163.4,164.2,171.7$; FAB-MS $m / z: 364$ $(\mathrm{M})^{+} ; \%$ Elemental anal. (found/calcd.): C 59.14/59.34; H 3.26/3.29; N 15.36/15.38.

5-Benzylidene-2-[\{5-(2-methylphenyl)-[1,3,4]-thiadiazol-2yl\} imino]-1,3-thiazolidin-4-one 4(b): $\mathrm{mp} 114$ - $116^{\circ} \mathrm{C}$; \% Yield: 71; IR (KBr) cm ${ }^{-1}$ : $3296(\mathrm{NH}), 1769(\mathrm{C}=\mathrm{O}), 1571(\mathrm{C}=\mathrm{N}) ;{ }^{1} \mathrm{H}$ NMR (300 MHz, DMSO- $\left.d_{6}\right) \delta 2.33\left(\mathrm{~s}, 3 \mathrm{H}, \mathrm{CH}_{3}\right), 7.10-7.36(\mathrm{~m}$, 9H, ArH), 7.42 (s, 1H, CH), 11.79 (bs, 1H, NH); \% Elemental anal. (found/calcd.): C 60.29/60.31, H 3.78/3.70, N 14.79/14.81.

5-Benzylidene-2-[\{5-(4-methylphenyl)-[1,3,4]-thiadiazol-2yl iminol-1,3-thiazolidin-4-one 4(c): $\mathrm{mp} 138$ - $139^{\circ} \mathrm{C}$; \% Yield: 69; IR (KBr) cm ${ }^{-1}$ : $3307(\mathrm{NH}), 1710(\mathrm{C}=\mathrm{O}), 1599(\mathrm{C}=\mathrm{N}) ;{ }^{1} \mathrm{H}$ NMR (300 MHz, DMSO- $\left.d_{6}\right) \delta 2.35\left(\mathrm{~s}, 3 \mathrm{H}, \mathrm{CH}_{3}\right), 7.12-7.38(\mathrm{~m}$, 9H, ArH), 7.46 (s, 1H, CH), 11.69 (bs, 1H, NH); \% Elemental anal. (found/calcd.): C 60.19/60.31; H 3.69/3.70; N 14.77/14.81.

5-Benzylidene-2-[\{5-(2-chlorophenyl)-[1,3,4]-thiadiazol-2yl\} imino]-1,3-thiazolidin-4-one 4(d): mp 122 - $124{ }^{\circ} \mathrm{C}$; \% Yield: 65; IR (KBr) cm ${ }^{-1}$ : $3357(\mathrm{NH}), 1754(\mathrm{C}=\mathrm{O}), 1597(\mathrm{C}=\mathrm{N}) ;{ }^{1} \mathrm{H}$ NMR (300 MHz, DMSO-d $) \delta$ 7.14-7.48 (m, 1H-CH, 9H-ArH), 12.19 (bs, 1H, NH); \% Elemental anal. (found/calcd.): C 54.05/ 54.20, H 2.75/2.76, N 13.99/14.05.

5-Benzylidene-2-[\{5-(4-chlorophenyl)-[1,3,4]-thiadiazol-2yl\} imino]-1,3-thiazolidin-4-one 4(e): mp 147 - $149{ }^{\circ} \mathrm{C}$; \% Yield: 78; IR (KBr) cm ${ }^{-1}$ : $3328(\mathrm{NH}), 1714(\mathrm{C}=\mathrm{O}), 1564(\mathrm{C}=\mathrm{N}) ;{ }^{1} \mathrm{H}$ NMR (300 MHz, DMSO-d $d_{6} \delta$ 7.16-7.52 (m, 1H-CH, 9H-ArH), 12.31 (bs, 1H, NH); \% Elemental anal. (found/calcd.): C 54.16/ 54.20, H 2.71/2.76, N 14.02/14.05.

5-Benzylidene-2-[\{5-(2-bromophenyl)-[1,3,4]-thiadiazol-2yl imino]-1,3-thiazolidin-4-one 4(f): $\mathrm{mp} 108-109{ }^{\circ} \mathrm{C}$; \% Yield: 61; IR (KBr) cm ${ }^{-1}$ : $3271(\mathrm{NH}), 1726(\mathrm{C}=\mathrm{O}), 1542(\mathrm{C}=\mathrm{N}) ;{ }^{1} \mathrm{H}$ NMR (300 MHz, DMSO-d $) \delta$ 7.14-7.49 (m, 1H-CH, 9H-ArH), 11.66 (bs, 1H, NH); \% Elemental anal. (found/calcd.): C 48.62/ 48.75, H 2.46/2.48, N 12.59/12.64.

5-Benzylidene-2-[\{5-(4-bromophenyl)-[1,3,4]-thiadiazol-2yl imino]-1,3-thiazolidin-4-one 4(g): $\mathrm{mp} 83-84{ }^{\circ} \mathrm{C}$; \% Yield: 68; IR (KBr) cm ${ }^{-1}$ : $3313(\mathrm{NH}), 1780(\mathrm{C}=\mathrm{O}), 1577(\mathrm{C}=\mathrm{N}) ;{ }^{1} \mathrm{H}$ NMR (300 MHz, DMSO- $d_{6}$ ) $\delta$ 7.20-7.58 (m, 1H-CH, 9H-ArH), 12.24 (bs, $1 \mathrm{H}, \mathrm{NH}, \mathrm{D}_{2} \mathrm{O}$-exchangeble); ${ }^{13} \mathrm{C}$ NMR $(75 \mathrm{MHz}$, DMSO- $\left.d_{6}\right) \delta 120.7,125.9,126.4,126.8,127.1,127.4,129.4$, 130.3, 136.5, 142.5, 154.3, 162.9, 163.4, 164.2, 171.7; FAB-MS $m / z: 443(\mathrm{M})^{+}, 445(\mathrm{M}+2)^{+} ; \%$ Elemental anal. (found/calcd.): C 48.73/48.75, H 2.46/2.48, N 12.63/12.64.

5-Benzylidene-2-[\{ 5-(3-nitrophenyl)-[1,3,4]-thiadiazol-2yl\} imino]-1,3-thiazolidin-4-one 4(h): $\mathrm{mp} 109-111^{\circ} \mathrm{C}$; \% Yield: 57; IR (KBr) cm ${ }^{-1}$ : $3374(\mathrm{NH}), 1737(\mathrm{C}=\mathrm{O}), 1568(\mathrm{C}=\mathrm{N}) ;{ }^{1} \mathrm{H}$ NMR (300 MHz, DMSO- $\left.d_{6}\right) \delta$ 6.69-7.30 (m, 9H, ArH), 7.37 (s, $1 \mathrm{H}, \mathrm{CH}), 11.26$ (bs, 1H, NH); \% Elemental anal. (found/calcd.): C 50.89/50.82, H 2.57/2.58, N 16.38/16.40.

5-Benzylidene-2-[\{ 5-(4-nitrophenyl)-[1,3,4]-thiadiazol-2- 
yl \} imino|-1,3-thiazolidin-4-one 4(i): $\mathrm{mp} 132$ - $135^{\circ} \mathrm{C}$; \% Yield: 53; IR (KBr) cm ${ }^{-1}: 3286(\mathrm{NH}), 1694(\mathrm{C}=\mathrm{O}), 1585(\mathrm{C}=\mathrm{N}) ;{ }^{1} \mathrm{H}$ NMR (300 MHz, DMSO- $\left.d_{6}\right) \delta$ 6.79-7.32 (m, 9H, ArH), 7.39 (s, $1 \mathrm{H}, \mathrm{CH}), 10.98$ (bs, 1H, NH); \% Elemental anal. (found/calcd.): C 50.86/50.82, H 2.52/2.58, N 16.38/16.40.

5-Benzylidene-2-[\{ 5-(2,4-dichlorophenyl)-[1,3,4]-thiadiazol-2-yl\} iminol-1,3-thiazolidin-4-one 4(j): $\mathrm{mp} 116-117^{\circ} \mathrm{C}$; \%Yield: 63; IR (KBr) cm ${ }^{-1}$ : $3358(\mathrm{NH}), 1729(\mathrm{C}=\mathrm{O}), 1547$ $(\mathrm{C}=\mathrm{N}) ;{ }^{1} \mathrm{H}$ NMR $\left(300 \mathrm{MHz}, \mathrm{DMSO}-d_{6}\right) \delta 7.24-7.51(\mathrm{~m}, 1 \mathrm{H}-\mathrm{CH}$, $8 \mathrm{H}-\mathrm{ArH}), 11.41$ (bs, 1H, NH); \% Elemental anal. (found/calcd.): C 45.81/45.88, H 2.27/2.30, N 12.89/12.93.

5-Benzylidene-2-[\{5-(2-hydroxyphenyl)-[1,3,4]-thiadiazol2-yl\} imino]-1,3-thiazolidin-4-one 4(k): mp 90 - $93{ }^{\circ} \mathrm{C}$; \% Yield: 79; IR (KBr) cm ${ }^{-1}$ : $3451(\mathrm{OH}), 3225(\mathrm{NH}), 1658(\mathrm{C}=\mathrm{O}), 1592$ $(\mathrm{C}=\mathrm{N}) ;{ }^{1} \mathrm{H}$ NMR $\left(300 \mathrm{MHz}, \mathrm{DMSO}-d_{6}\right) \delta 6.96-7.37(\mathrm{~m}, 1 \mathrm{H}-\mathrm{CH}$, 9H-ArH), 9.41 (s1H, OH), 11.29 (bs, 1H, NH); \% Elemental anal. (found/calcd.): C 56.81/56.84, H 3.10/3.15, N 14.71/14.73.

5-Benzylidene-2-[\{5-(4-methoxyphenyl)-[1,3,4]-thiadiazol2-yl\} imino]-1,3-thiazolidin-4-one 4(I): $\mathrm{mp} 98-100{ }^{\circ} \mathrm{C}$; \% Yield: 67; IR (KBr) cm ${ }^{-1}$ : $3347(\mathrm{NH}), 1722(\mathrm{C}=\mathrm{O}), 1573(\mathrm{C}=\mathrm{N}) ;{ }^{1} \mathrm{H}$ NMR (300 MHz, DMSO-d $\left.d_{6}\right) \delta 3.74\left(\mathrm{~s}, 3 \mathrm{H}, \mathrm{OCH}_{3}\right), 6.84-7.53$ (m, 1H-CH, 9H-ArH), 11.88 (bs, 1H, NH); \% Elemental anal. (found/calcd.): C 57.82/57.86, H 3.54/3.55, N 14.20/14.21.

General Method for Synthesis of 2-[\{5-(Substituted phenyl)$[1,3,4]$-thiadiazol-2-yl $\}$ imino]-5-(3,4-dimethoxybenzylidene)1,3-thiazolidin-4-one 5(a-l). To the compound 3 (a-l) (3 mmol) in $20 \mathrm{~mL}$ ethanol, $2 \mathrm{~mL}$ piperidine and $3 \mathrm{mmol}$ of 3, 4-dimethoxy benzaldehyde was added. The mixture was refluxed for 12 $18 \mathrm{hrs}$. The mixture was poured over crushed ice and the solution was neutralized with $\mathrm{HCl}$. The precipitate was filtered, washed with water, dried and recrystallized from ethanol-DMF to yield final compound 5(a-l). The purity of compound was analyzed by TLC using benzene: acetone (9:1) as mobile phase.

2-[\{5-Phenyl-[1,3,4]-thiadiazol-2-yl $\}$ imino]-5-(3,4-dimethoxybenzylidene)-1,3-thiazolidin-4-one 5(a): $\mathrm{mp} 113-115^{\circ} \mathrm{C}$; \%Yield: 68; IR (KBr) cm ${ }^{-1}$ : $3352(\mathrm{NH}), 1743(\mathrm{C}=\mathrm{O}), 1546$ $(\mathrm{C}=\mathrm{N}) ;{ }^{1} \mathrm{H}$ NMR $\left(300 \mathrm{MHz}, \mathrm{DMSO}-d_{6}\right) \delta 3.86\left(\mathrm{~s}, 6 \mathrm{H}, 2 \mathrm{xOCH}_{3}\right)$, 6.84-7.93 (m, 1H-CH, 8H-ArH), 12.86 (bs, 1H, NH, D 2 exchangeble); ${ }^{13} \mathrm{C}$ NMR (75 MHz, DMSO- $\left.d_{6}\right) \delta 56.3,115.4$, 112.4, 119.6, 126.8, 127.1, 128.4, 129.4, 136.5, 142.5, 143.5, 146.3, 154.2, 163.7, 164.2, 172.7; FAB-MS $m / z: 424(\mathrm{M})^{+}, 425$ $(\mathrm{M}+1)^{+} ; \%$ Elemental anal. (found/calcd.): C 56.56/56.60, H 3.74/3.77, N 13.23/13.21.

2-[\{5-(2-Methylphenyl)-[1,3,4]-thiadiazol-2-yl $\}$ imino $]-5-$ (3,4-dimethoxybenzylidene)-1,3-thiazolidin-4-one 5(b): $\mathrm{mp}$ 96 - $99{ }^{\circ} \mathrm{C}$; \% Yield: 74; IR (KBr) cm ${ }^{-1}: 3330(\mathrm{NH}), 1738(\mathrm{C}=\mathrm{O})$, $1556(\mathrm{C}=\mathrm{N}) ;{ }^{1} \mathrm{H}$ NMR $\left(300 \mathrm{MHz}, \mathrm{DMSO}-d_{6}\right) \delta 2.35(\mathrm{~s}, 3 \mathrm{H}$, $\left.\mathrm{CH}_{3}\right), 3.73$ (s, $6 \mathrm{H}, 2 \mathrm{xOCH}_{3}$ ), 7.26-8.20 (m, 1H-CH, 7H-ArH), 11.68 (bs, $1 \mathrm{H}, \mathrm{NH}) ; \%$ Elemental anal. (found/calcd.): C 57.49/ 57.53, H 4.13/4.11, N 12.72/12.79.

2-[\{5-(4-Methylphenyl)-[1,3,4]-thiadiazol-2-yl\} imino]-5(3,4-dimethoxybenzylidene)-1,3-thiazolidin-4-one 5(c): $\mathrm{mp}$ 91 - $93{ }^{\circ} \mathrm{C}$; \% Yield: 59; IR (KBr) cm ${ }^{-1}: 3287(\mathrm{NH}), 1699(\mathrm{C}=\mathrm{O})$, $1523(\mathrm{C}=\mathrm{N}) ;{ }^{1} \mathrm{H}$ NMR (300 MHz, DMSO-d $) \delta 2.32(\mathrm{~s}, 3 \mathrm{H}$, $\mathrm{CH}_{3}$ ), 3.81 (s, 6H, 2xOCH 3 ), 7.16-7.98 (m, 1H-CH, 7H-ArH), 12.34 (bs, $1 \mathrm{H}, \mathrm{NH}) ; \%$ Elemental anal. (found/calcd.): C 57.58/ 57.53, H 4.11/4.11, N 12.74/12.79.

2-[\{5-(2-Chlorophenyl)-[1,3,4]-thiadiazol-2-yl\} imino]-5- (3,4-dimethoxybenzylidene)-1,3-thiazolidin-4-one 5(d): $\mathrm{mp}$ 110 - $112{ }^{\circ} \mathrm{C}$; \%Yield: 64; IR (KBr) cm ${ }^{-1}: 3316(\mathrm{NH}), 1777$ $(\mathrm{C}=\mathrm{O}), 1525(\mathrm{C}=\mathrm{N}) ;{ }^{1} \mathrm{H}$ NMR $\left(300 \mathrm{MHz}, \mathrm{DMSO}-d_{6}\right) \delta 3.58(\mathrm{~s}$, $6 \mathrm{H}, 2 \mathrm{xOCH}_{3}$ ), 7.37-8.06 (m, 1H-CH, 7H-ArH), 10.44 (bs, 1H, $\mathrm{NH}$ ); \% Elemental anal. (found/calcd.): C 52.31/52.34, H 3.25/ $3.27, \mathrm{~N} 12.19 / 12.21$.

2-[\{5-(4-Chlorophenyl)-[1,3,4]-thiadiazol-2-yl\} imino]-5(3,4-dimethoxybenzylidene)-1,3-thiazolidin-4-one 5(e): $\mathrm{mp}$ 104 - $106{ }^{\circ} \mathrm{C}$; \%Yield: 72; IR (KBr) cm ${ }^{-1}$ : $3288(\mathrm{NH}), 1762$ $(\mathrm{C}=\mathrm{O}), 1541(\mathrm{C}=\mathrm{N}) ;{ }^{1} \mathrm{H}$ NMR $\left(300 \mathrm{MHz}, \mathrm{DMSO}-d_{6}\right) \delta 3.64(\mathrm{~s}$, $\left.6 \mathrm{H}, 2 \mathrm{xOCH}_{3}\right), 7.41-8.18$ (m, 1H-CH, 7H-ArH), 12.04 (bs, 1H, $\mathrm{NH}$ ); \% Elemental anal. (found/calcd.): C 52.29/52.34, H3.21/ 3.27, N 12.18/12.21.

2-[\{5-(2-Bromophenyl)-[1,3,4]-thiadiazol-2-yl $\}$ imino]-5(3,4-dimethoxybenzylidene)-1,3-thiazolidin-4-one 5(f): $\mathrm{mp}$ 85 - $87^{\circ} \mathrm{C}$; \% Yield: 76; IR (KBr) cm ${ }^{-1}: 3328(\mathrm{NH}), 1728(\mathrm{C}=\mathrm{O})$, $1553(\mathrm{C}=\mathrm{N}) ;{ }^{1} \mathrm{H}$ NMR $\left(300 \mathrm{MHz}, \mathrm{DMSO}-d_{6}\right) \delta 3.42(\mathrm{~s}, 6 \mathrm{H}$, $2 \mathrm{xOCH}_{3}$ ), 7.22-7.89 (m, 1H-CH, 7H-ArH), 11.62 (bs, 1H, NH); $\%$ Elemental anal. (found/calcd.): C 47.67/47.71, H 2.94/2.98, N 11.09/11.13.

2-[\{5-(4-Bromophenyl)-[1,3,4]-thiadiazol-2-yl $\}$ imino $]-5-$ (3,4-dimethoxybenzylidene)-1,3-thiazolidin-4-one 5(g): $\mathrm{mp}$ 79 - $81{ }^{\circ} \mathrm{C}$; \% Yield: 84; IR (KBr) cm ${ }^{-1}: 3280(\mathrm{NH}), 1698(\mathrm{C}=\mathrm{O})$, $1518(\mathrm{C}=\mathrm{N})$ ) ${ }^{1} \mathrm{H}$ NMR $\left(300 \mathrm{MHz}, \mathrm{DMSO}-d_{6}\right) \delta 3.79(\mathrm{~s}, 6 \mathrm{H}$, $2 \mathrm{xOCH}_{3}$ ), 7.28-7.99 (m, 1H-CH, 7H-ArH), 11.39 (bs, $1 \mathrm{H}, \mathrm{NH}$, $\mathrm{D}_{2} \mathrm{O}$-exchangeble); \% Elemental anal. (found/calcd.): C 47.68/ 47.71, H 2.91/2.98, N 11.11/11.13.

2-[\{5-(3-Nitrophenyl)-[1,3,4]-thiadiazol-2-yl\} imino]-5(3,4-dimethoxybenzylidene)-1,3-thiazolidin-4-one 5(h): $\mathrm{mp}$ 85 - $86^{\circ} \mathrm{C}$; \% Yield: 77; IR (KBr) cm ${ }^{-1}: 3298(\mathrm{NH}), 1767(\mathrm{C}=\mathrm{O})$, $1561(\mathrm{C}=\mathrm{N}) ;{ }^{1} \mathrm{H}$ NMR $\left(300 \mathrm{MHz}, \mathrm{DMSO}-d_{6}\right) \delta 3.82(\mathrm{~s}, 6 \mathrm{H}$, $2 \mathrm{xOCH}_{3}$ ), 7.39-8.62 (m, 1H-CH, 7H-ArH), 10.84 (bs, 1H, NH); $\%$ Elemental anal. (found/calcd.): C 49.47/49.48, H 3.02/3.09, N 14.42/14.43.

2-[\{5-(4-Nitrophenyl)-[1,3,4]-thiadiazol-2-yl $\}$ imino]-5(3,4-dimethoxybenzylidene)-1,3-thiazolidin-4-one 5(i): $\mathrm{mp}$ 94 - $96^{\circ} \mathrm{C}$; \% Yield: 67; IR (KBr) cm ${ }^{-1}: 3284(\mathrm{NH}), 1700(\mathrm{C}=\mathrm{O})$, $1553(\mathrm{C}=\mathrm{N}) ;{ }^{1} \mathrm{H}$ NMR $\left(300 \mathrm{MHz}, \mathrm{DMSO}-d_{6}\right) \delta 3.86(\mathrm{~s}, 6 \mathrm{H}$, $2 \mathrm{xOCH}_{3}$ ), 7.27-7.86 (m, 1H-CH, 7H-ArH), 10.21 (bs, 1H, NH); $\%$ Elemental anal. (found/calcd.): C 49.48/49.48, H 3.09/3.09, N 14.39/14.43.

2-[\{5-(2,4-Dichlorophenyl)-[1,3,4]-thiadiazol-2-yl $\}$ imino]5-(3,4-dimethoxybenzylidene)-1,3-thiazolidin-4-one 5(j): $\mathrm{mp}$ $117-120{ }^{\circ} \mathrm{C}$; \%Yield: 71; IR (KBr) cm $\mathrm{cm}^{-1}: 3348(\mathrm{NH}), 1717$ $(\mathrm{C}=\mathrm{O}), 1542(\mathrm{C}=\mathrm{N}) ;{ }^{1} \mathrm{H}$ NMR $\left(300 \mathrm{MHz}, \mathrm{DMSO}-d_{6}\right) \delta 3.51$ (s, 6H, 2xOCH 3 ), 7.42-8.28 (m, 1H-CH, 6H-ArH), 11.64 (bs, $1 \mathrm{H}, \mathrm{NH}$ ); \% Elemental anal. (found/calcd.): C 48.70/48.68, H 2.83/2.84, N 11.34/11.36.

2-[\{5-(2-Hydroxyphenyl)-[1,3,4]-thiadiazol-2-yl $\}$ imino]5-(3,4-dimethoxybenzylidene)-1,3-thiazolidin-4-one 5(k): $\mathrm{mp}$ 79 - $80{ }^{\circ} \mathrm{C}$; \% Yield: 82; IR (KBr) cm ${ }^{-1}: 3418(\mathrm{OH}), 3309(\mathrm{NH})$, $1726(\mathrm{C}=\mathrm{O}), 1565(\mathrm{C}=\mathrm{N}) ;{ }^{1} \mathrm{H}$ NMR $\left(300 \mathrm{MHz}, \mathrm{DMSO}-d_{6}\right) \delta$ $3.23\left(\mathrm{~s}, 6 \mathrm{H}, 2 \mathrm{xOCH}_{3}\right), 7.22-7.98(\mathrm{~m}, 1 \mathrm{H}-\mathrm{CH}, 7 \mathrm{H}-\mathrm{ArH}), 9.43$ (s, $1 \mathrm{H}, \mathrm{OH}), 12.15$ (bs, $1 \mathrm{H}, \mathrm{NH}) ; \%$ Elemental anal. (found/calcd.): C 54.51/54.55, H 3.58/3.64, N 12.71/12.73.

2-[\{5-(4-Methoxyphenyl)-[1,3,4]-thiadiazol-2-yl\} imino]5-(3,4-dimethoxybenzylidene)-1,3-thiazolidin-4-one 5(I): $\mathrm{mp}$ 157 - $158{ }^{\circ} \mathrm{C}$; \%Yield: 54; IR (KBr) cm ${ }^{-1}$ : 3322 (NH), 1678 
$(\mathrm{C}=\mathrm{O}), 1524(\mathrm{C}=\mathrm{N}) ;{ }^{1} \mathrm{H}$ NMR $\left(300 \mathrm{MHz}, \mathrm{DMSO}-d_{6}\right) \delta 3.61(\mathrm{~s}$, $9 \mathrm{H}, 3 \mathrm{xOCH}_{3}$ ), 7.19-7.63 (m, 1H-CH, 7H-ArH), 10.92 (bs, $1 \mathrm{H}, \mathrm{NH}$ ); \% Elemental anal. (found/calcd.): C 55.33/55.38, H 4.16/4.18, N 12.34/12.31.

The above synthesized compounds 5-(substituted-arylidene)2-[\{5-(substituted-phenyl)-(1,3,4)-thiadiazol-2-yl $\}$ imino $]-1,3-$ thiazolidin-4-one [4(a-l) \& 5(a-l)] were then screened for their anti-convulsant activity and neurotoxicity studies by the maximal electroshock (MES) ${ }^{2,3}$ induced seizures method and rotarod method $^{4}$ respectively.

Maximal Electroshock Test (MES test). This test was done on swiss albino mice $(25-30 \mathrm{~g})$ in groups of 6 each. The test compounds were administered as an i.p. injection at dose level of 30,100 or $300 \mathrm{mg} / \mathrm{kg}$ b.w. and the anticonvulsant activity was assessed after $0.5 \mathrm{hr}$. and $4 \mathrm{hrs}$. intervals of administration. Maximal electroshock seizures were elicited in mice by delivering $60 \mathrm{~Hz}, 50 \mathrm{~mA}$ electrical stimuli for $0.2 \mathrm{~s}$ via ear clip electrodes. The maximal seizure typically consists of a short period of tonic extension of the hind limbs and a final clonic episode. Blockade of the hind limbs tonic extensor component due to the drug treatment is taken as the end point. Phenytoin sodium, $30 \mathrm{mg} / \mathrm{kg}$ b.w. was used as the standard drug for evaluation.

Rotarod Test. Rotarod test has been performed to detect the minimal motor deficit in mice. Swiss albino mice (25 - $30 \mathrm{~g})$ were used in groups of 6 each and trained to stay on an accelerating rotarod that rotates at $10 \mathrm{rpm}$. The rod diameter was $3.2 \mathrm{~cm}$ in diameter. Trained animals (able to stay on the rotarod for at least two consecutive trials of 90 s each) were given an i.p. injection of the test compounds at doses $30,100,300 \mathrm{mg} / \mathrm{kg} \mathrm{b.w}$. Neurological deficit was indicated by the inability of the animal to maintain equilibrium on the rod for at least $1 \mathrm{~min}$ in each of the three trials. The test compounds were compared with phenytoin as standard at dose of $30 \mathrm{mg} / \mathrm{kg}$ b.w.

\section{Result and Discussion}

Various substituted benzoic acids were initially treated with thiosemicarbazide in presence of cyclizing agent $\mathrm{POCl}_{3}$ to give compounds $\mathbf{1}(\mathbf{a}-\mathbf{l}){ }^{5}$ The compounds $\mathbf{1}(\mathbf{a}-\mathbf{l})$ were then treated with chloroacetylchloride to produce 2-chloro- $N$-[5-(substitutedphenyl $[1,3,4]$ thiadiazol-2-yl)]-acetamide 2(a-l) and was characterized by an additional singlet of $\mathrm{CH}_{2}$ in ${ }^{1} \mathrm{H}$ NMR spectra. Further, 2(a-I) was reacted with ammoniumthiocyanate to give cyclized compounds $\mathbf{3 ( a - 1 )}{ }^{6}$ and the singlet for cyclized $\mathrm{CH}_{2}$ shows an upfield shift which was then subjected to Knoevenagel condensation with benzaldehyde and 3,4 dimethoxybenzaldehyde to yield final compounds as 5-benzylidene 2-[5-(substi-
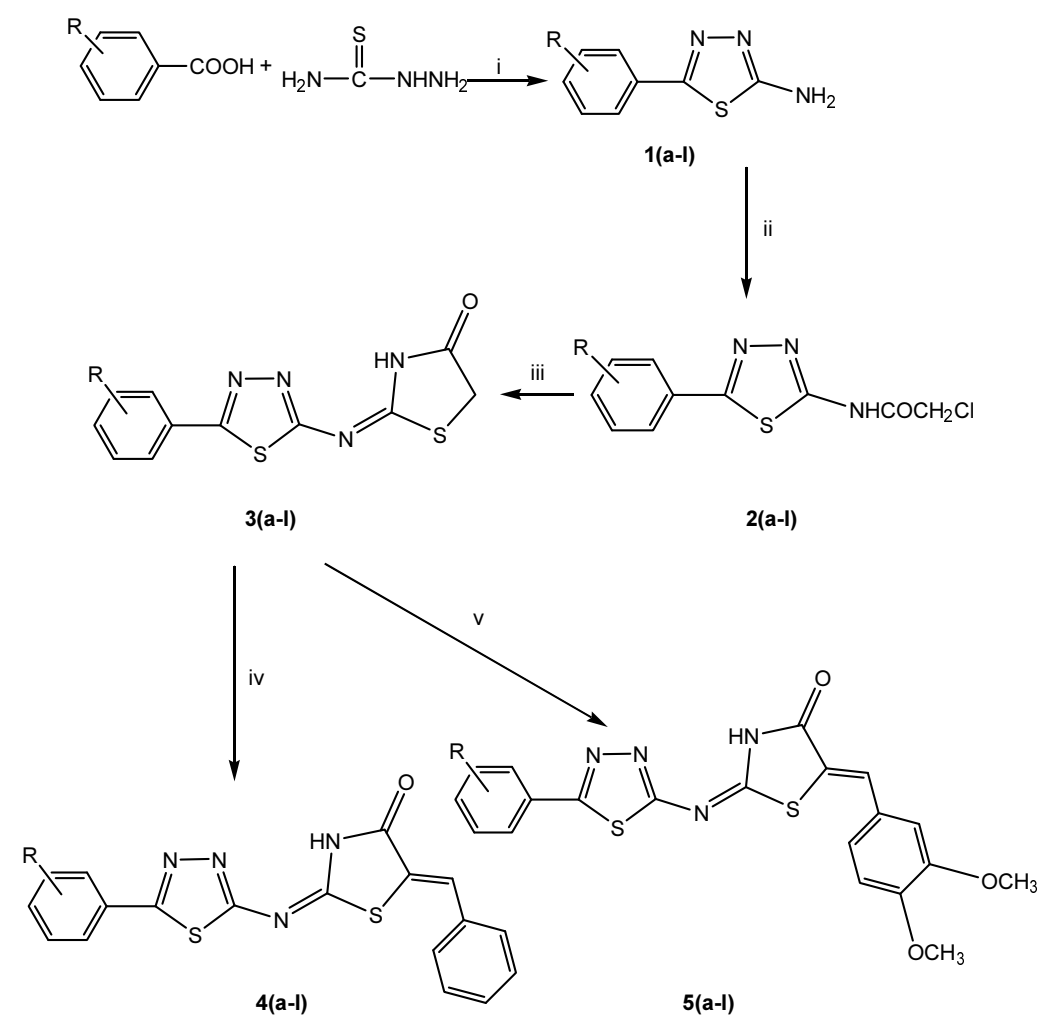

\begin{tabular}{|c|c|c|c|c|c|c|c|c|c|c|c|c|}
\hline $1,2,3,4,5$ & $\mathbf{a}$ & b & c & d & e & f & g & h & i & $\mathbf{j}$ & $\mathbf{k}$ & 1 \\
\hline $\mathrm{R}$ & $\mathrm{H}$ & $2-\mathrm{CH}_{3}$ & $4-\mathrm{CH}_{3}$ & $2-\mathrm{Cl}$ & $4-\mathrm{Cl}$ & $2-\mathrm{Br}$ & $4-\mathrm{Br}$ & $3-\mathrm{NO}_{2}$ & $4-\mathrm{NO}_{2}$ & $2,4-\mathrm{Cl}$ & $2-\mathrm{OH}$ & $4-\mathrm{OCH}_{3}$ \\
\hline
\end{tabular}

Scheme 1. (i) $\mathrm{POCl}_{3}$, reflux; (ii) $\mathrm{ClCOCH}_{2} \mathrm{Cl}$, dry pyridine, dry benzene, reflux; (iii) $\mathrm{NH}_{4} \mathrm{SCN}$, ethanol, reflux; (iv) benzaldehyde, piperidine, absolute ethanol; (v) 3,4-dimethoxy benzaldehyde, piperidine, absolute ethanol 
Table 1. Anticonvulsant and Neurotoxicity data for compounds 4(a-l) \& 5(a-l)

\begin{tabular}{ccccc}
\hline \multirow{2}{*}{ Compound } & \multicolumn{2}{c}{ MES screening } & \multicolumn{2}{c}{ Neurotoxicity screening } \\
\cline { 2 - 5 } & 0.5 hr. & 4 hr. & 0.5 hr. & 4 hr. \\
\hline $\mathbf{4 a}$ & 30 & 100 & - & - \\
$\mathbf{4 b}$ & 100 & 300 & 100 & 300 \\
$\mathbf{4 c}$ & 100 & 300 & 300 & - \\
$\mathbf{4 d}$ & 100 & 100 & 300 & - \\
$\mathbf{4 e}$ & 30 & 100 & 300 & - \\
$\mathbf{4 h}$ & 300 & - & 300 & - \\
$\mathbf{4 i}$ & 300 & - & - & - \\
$\mathbf{4 j}$ & 30 & 100 & 300 & - \\
$\mathbf{4 k}$ & 100 & - & 100 & 300 \\
$\mathbf{4 l}$ & 100 & 300 & 300 & 300 \\
$\mathbf{5 a}$ & 100 & 100 & - & - \\
$\mathbf{5 b}$ & 100 & 300 & 100 & 300 \\
$\mathbf{5 c}$ & 100 & 100 & 100 & - \\
$\mathbf{5 d}$ & 100 & 100 & 300 & - \\
$\mathbf{5 e}$ & 100 & 100 & 300 & - \\
$\mathbf{5 h}$ & 300 & - & 300 & - \\
$\mathbf{5 i}$ & 300 & - & 300 & - \\
$\mathbf{5 j}$ & 30 & 100 & 300 & - \\
$\mathbf{5 k}$ & 100 & - & 100 & 300 \\
$\mathbf{5 l}$ & 100 & 300 & 100 & 100 \\
Phenytoin & 30 & 30 & 100 & 100 \\
\hline & & & &
\end{tabular}

Doses of 30,100 and $300 \mathrm{mg} / \mathrm{kg}$ of the compound were administered and the protection and neurotoxicity measured after $0.5 \mathrm{hr}$. and $4 \mathrm{hrs}$. The data indicate the minimal dose required to cause protection or neurotoxicity in $50 \%$ or more of the animals. The dash (-) indicates the absence of anticonvulsant activity or neurotoxicity.

tuted-phenyl $[1,3,4]$-thiadiazol-2-ylimino) thiazolidin-4-one 4(a-l) and 2-[5-(substituted-phenyl [1,3,4]-thiadiazol-2-ylimino)-5-(3,4-dimethoxy benzylidene) thiazolidin-4-one 5(a-l) respectively (Scheme 1).

The results of anticonvulsant and neurotoxicity screening for the final compounds of Scheme 1 are summarized in Table 1. The anticonvulsant activity for the compounds was carried out by MES model followed by screening the same compounds for their neurotoxic effect. Data is presented as minimal dose required causing protection against seizers or neurotoxicity in $50 \%$ or more of the animals. Doses of $30,100,300 \mathrm{mg} / \mathrm{kg}$ of the compound were administered and the protection against seizers and neurotoxicity both were measured after $0.5 \mathrm{hr}$. and $4 \mathrm{hr}$. Phenytoin was used as standard for the MES test.

Among tested thiadiazole-thiazolidinone derivatives, most of the compounds showed good anticonvulsant activity indicative of their ability to prevent seizure spread at dose of 30 , $100,300 \mathrm{mg} / \mathrm{kg}$. The compounds $\mathbf{4 a}, \mathbf{4 e}, \mathbf{4 j}$ and $\mathbf{5 j}$ prevented seizures at dose of 30 and $100 \mathrm{mg} / \mathrm{kg}$ at the end of $0.5 \mathrm{hr}$. and $4 \mathrm{hrs}$. Few of them (mainly h \& i derivates) demonstrated activity only at $300 \mathrm{mg} / \mathrm{kg}$ dose while rest of the compounds prevented seizures at $100 \mathrm{mg} / \mathrm{kg}$ after time interval of half an hour of drug administration.

The general trends may be discerned from the data of MES screening that $60 \%$ of the compounds had greater activity at the end of $0.5 \mathrm{hr}$. than after $4 \mathrm{hrs}$. Thus in general, these compounds are short acting anticonvulsant. Secondly, protection was afforded by all the tested compounds in the MES screening.

As observed through data, all the tested compounds of the series with varied substitution portrayed anticonvulsant activity. The unsubstituted compounds (4a) and the compounds with electronegative substituents $(\mathbf{4 e}, \mathbf{4 j} \boldsymbol{\mathbf { S }} \mathbf{5} \mathbf{j})$ were found to be more potent when compared to compounds containing electron releasing groups. Furthermore, on comparing the data for different derivatives of 4th and 5th product of the Scheme 1, the derivatives of 4th product showed comparatively better activity than derivatives of 5th suggesting that the presence of methoxy group being electron releasing showed less activity. Besides this, the neurotoxicity screening of compounds revealed that most of the compounds were neurotoxic at anticonvulsant dose. Only compounds without substitution at the phenyl ring i.e. $4 \mathbf{a} \& 5 \mathbf{a}$ did not show neurotoxicity at maximum administered dose of $300 \mathrm{mg} / \mathrm{kg}$.

Acknowledgments. The authors acknowledge the financial support of the University Grant Commission, India and Jamia Hamdard for providing laboratory and other basic facilities for carrying out experimental work.

\section{References}

1. Pandeya, S. N.; Raja, A. S.; Stables, J. P. J. Pharm. Pharmaceut Sci. 2002, 5(3), 266.

2. Krall, R. L.; Penry, J. K.; White, B. G.; Kupferberg, H. J.; Swinyard, E. A. Epilepsia 1978, 19, 409.

3. White, H. S.; Johnson, M.; Wolf, H. H.; Kupferberg, H. J. Ital. J. Sci. 1995, 16, 73.

4. Dunham, M. S.; Miya, T. A. J. Am. Pharmac. Assoc. Sci. Edit. 1957, 46, 208

5. Shafiee, A.; Naimi, E.; Mansobi, P.; Foroumadi, A.; Shekari, M. J. Het. Chem. 1995, 32, 1235.

6. Altıntaş, H.; Ateş, O.; Kocabalkanli, A.; Birteksoz, S.; Otuk, G. Ind. J. Chem. 2005, 44B, 585. 